\title{
Three concurrent variations of the aberrant right subclavian artery, the non-recurrent laryngeal nerve and the right thoracic duct
}

\author{
J.-Y. Lee, D.-Y. Won, S.-H. Oh, S.-Y. Hong, R.-S. Woo, T.-K. Baik, H.-I. Yoo*, D.-Y. Song* \\ Department of Anatomy and Neuroscience, Eulji University School of Medicine, Daejeon, Republic of Korea
}

[Received: 15 February 2016; Accepted: 5 April 2016]

\begin{abstract}
We herein report a case showing three anatomical variations including the aberrant right subclavian artery (ARSA), the non-recurrent laryngeal nerve (NRLN) and the right thoracic duct in a 59-year-old male cadaver. The right subclavian artery (RSA) arose from the descending aorta next to the left subclavian artery and coursed in between the oesophagus and the thoracic vertebrae. The recurrent laryngeal nerve did not coil around the RSA but directly entered the larynx. Lastly the thoracic duct terminated into the right brachiocephalic vein. This study makes an embryological assumption that the abnormal development of the RSA had happened first and subsequently caused NRLN and the thoracic duct drainage variation. As to our knowledge, only two reports have been made previously concerning such concurrent variations. Therefore, this case report alerts anatomists and clinicians to the possibility of simultaneous occurrence of ARSA, NRLN and the right thoracic duct. (Folia Morphol 2016; 75, 4: 560-564)
\end{abstract}

Key words: aberrant right subclavian artery, dysphagia lusoria, non-recurrent laryngeal nerve, retro-oesophageal right subclavian artery, right thoracic duct

\section{INTRODUCTION}

In the majority of population, the right subclavian artery (RSA) originates from the brachiocephalic trunk, the first branch of the aortic arch, along with the right common carotid artery [16]. As the name suggests, it arises deep to the right sternoclavicular joint, ascends over the clavicle and terminates medial to the first rib and becomes the axillary artery. Along its course the recurrent laryngeal nerve interferes. The right recurrent laryngeal nerve coils around the RSA and courses along the tracheo-oesophageal groove [20]. The nerve then passes the inferior thyroid artery and innervates the lower larynx and branches of the trachea, oesophagus and the inferior constrictor muscles.
As for the thoracic duct, it originates from the cisterna chyli and passes through the aortic hiatus, entering the posterior mediastinum. The thoracic duct lies to the right side of the thoracic vertebrae up to the $5^{\text {th }}$ thoracic vertebra. Then it crosses over the vertebral column continuing upwards and emptying into the venous system, the site of the left jugular and subclavian veins, and sometimes into the left brachiocephalic vein.

In a male cadaver dissected in Eulji University in South Korea, however, the RSA, the recurrent laryngeal nerve and the thoracic duct all showed anatomical difference from the ordinary course. The present report describes three concurrent variations,

Address for correspondence: Dr D.-Y. Song, Department of Anatomy and Neuroscience, Eulji University School of Medicine, 301-832, 143-5, Yongdu-dong, Jung-gu, Daejeon, Republic of Korea, tel: +82-42-259-1622, fax: +82-42-259-1629, e-mail: dysong@eulji.ac.kr *H.-I.Yoo and D.-Y. Song equally contributed as the corresponding authors. 

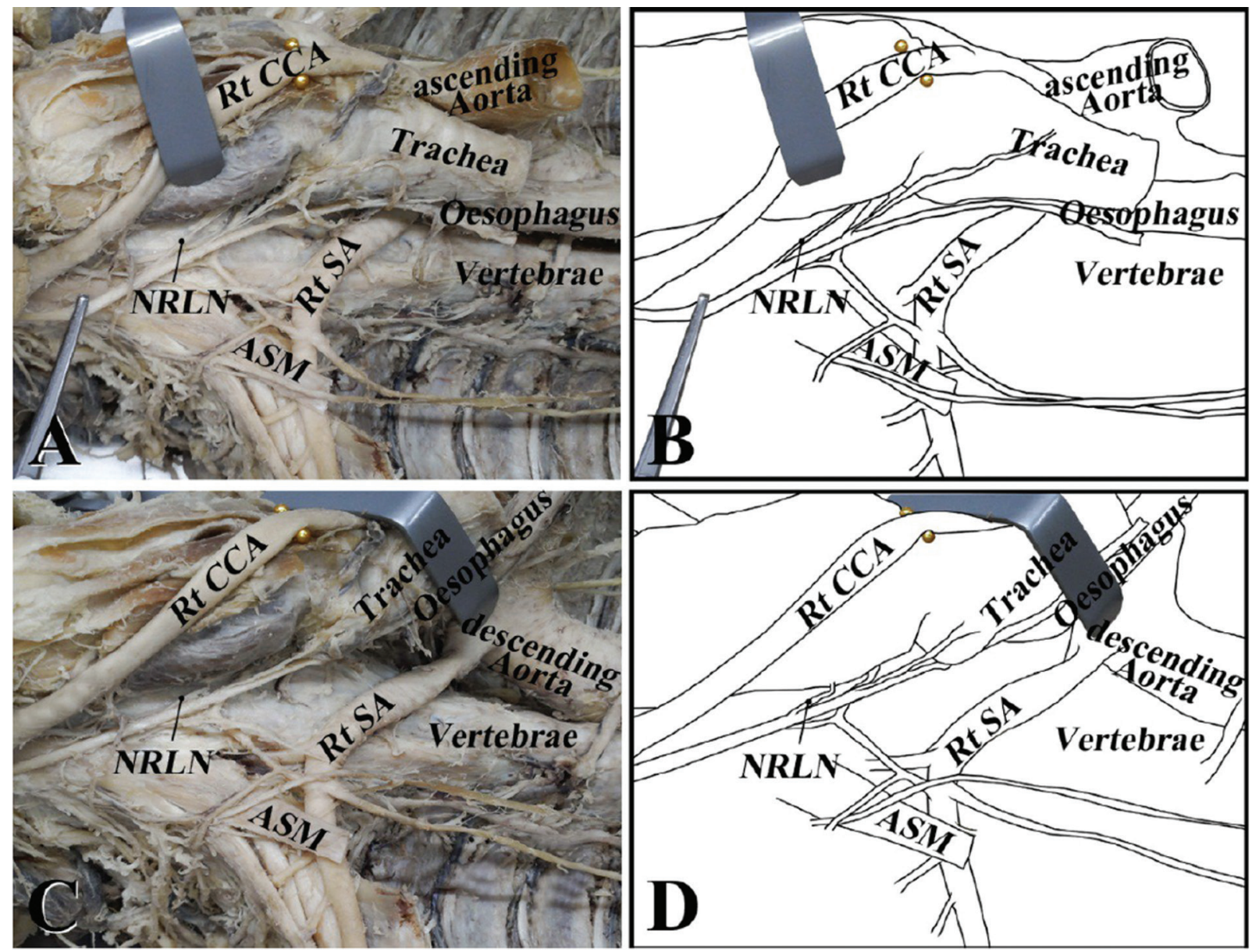

Figure 1. A. The right subclavian artery (Rt SA) passes in between the oesophagus and the vertebrae; $B$. Schematic diagram of panel A; C. Ascending aorta, trachea and oesophagus are retracted for a better view of the Rt SA arising from the descending aorta; D. Schematic diagram of panel C; Rt CCA — right common carotid artery; NRLN — non-recurrent laryngeal nerve; ASM — anterior scalenus muscle.

speculates the possible embryological background and highlights their clinical importance.

\section{CASE REPORT}

In Eulji University during a gross anatomy class, a male cadaver dissected had three anatomical variations including the aberrant right subclavian artery (ARSA), the non-recurrent laryngeal nerve (NRLN) and the right thoracic duct. From 1999 to 2015, approximately 166 cadaver dissections were performed in the university and this is the first instance of such simultaneous variations. The male cadaver is of Korean nationality and lived from 1945 to 2014. According to his medical record, he suffered from multiple sclerosis during his lifetime and passed away due to cardiac arrest. The photos were photographed using a Samsung digital camera (VLUU NV10: Samsung Inc., Seoul, Korea) and schematic diagrams were illustrated using Adobe Photoshop (version 7.0: Adobe System Inc., San Jose, CA, USA).
In cadaver dissection we found the RSA originating from the descending aorta as the $4^{\text {th }}$ branch following the right and the left common carotid artery and the left subclavian artery. It then ascended obliquely between the oesophagus and the thoracic vertebrae (Fig. 1). Hence, ARSA of this case was retrooesophageal. Despite its aberrant origin, the branches of the artery seemed ordinary: the vertebral artery just after ARSA passes below the larynx, the thyrocervical trunk and the internal thoracic artery medial to the scalenus anterior, the right costocervical artery deep to the muscle, and finally the transverse cervical and the suprascapular artery lateral to the muscle. As for the right recurrent laryngeal nerve, after arising from the vagus nerve, it did not coil around RSA but directly entered the larynx thus becoming the NRLN (Fig. 2A). During its course, the nerve passed anterior and parallel to the inferior thyroid artery and superior to the external branch of the superior laryngeal nerve. 

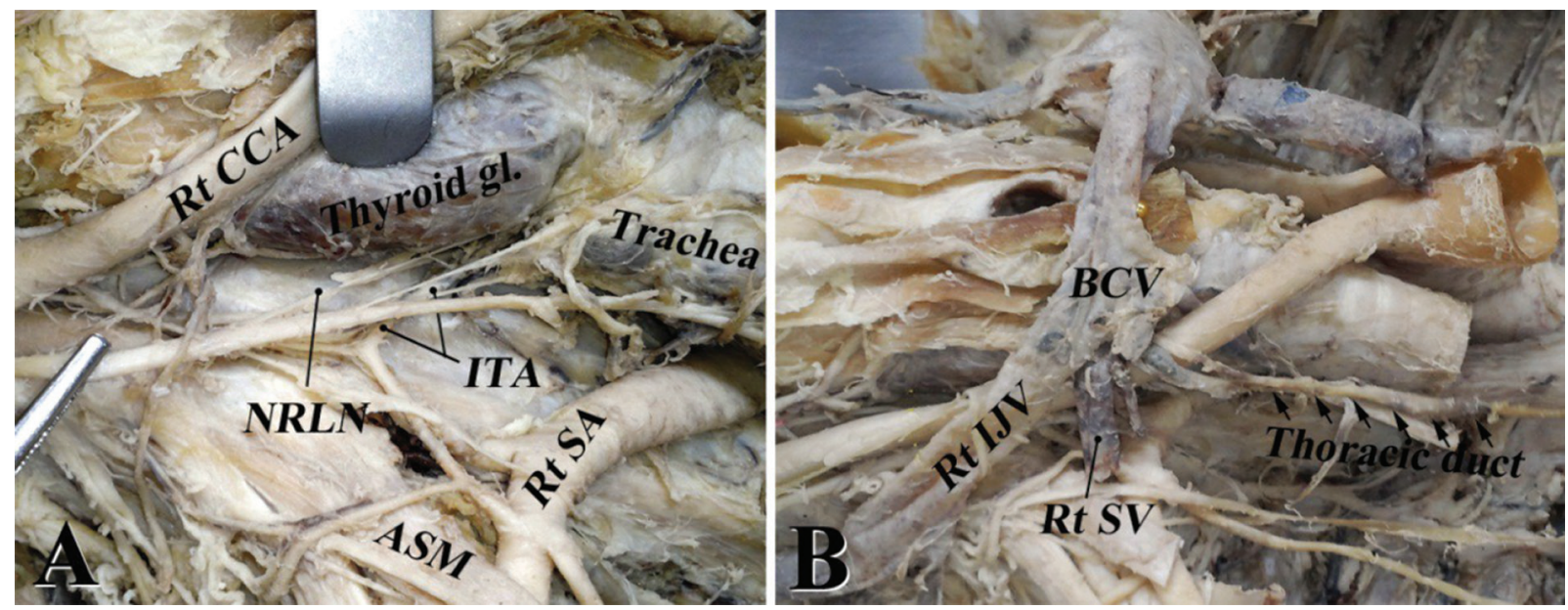

Figure 2. A. The non-recurrent laryngeal nerve courses anterior and parallel to the inferior thyroid artery (ITA) without coiling around the right subclavian artery (Rt SA); B. The thoracic duct terminates at the right brachiocephalic vein (BCV); Rt CCA — right common carotid artery; NRLN — non-recurrent laryngeal nerve; ASM — anterior scalenus muscle; Rt IJV — right internal jugular vein; Rt SV — right subclavian vein.

Concerning the thoracic duct, in the present cadaver, the origin of the duct was normal as it derived from the cisterna chyli and passed through the aortic hiatus to enter the posterior mediastinum. However, it then took an aberrant course as it ascended between the thoracic aorta and the azygos vein and terminated into the right brachiocephalic vein (Fig. 2B). Therefore, the thoracic duct in this case drained to the right side of the vein.

\section{DISCUSSION}

Aberrant right subclavian artery is reported to exist in $0.5-1.8 \%$ of the population [4]. ARSA may arise rarely as the second or the $3^{\text {rd }}$ branch of the aortic arch, but mostly as the $4^{\text {th }}$. According to Quain [16], ARSA may take three courses: more commonly in between the oesophagus and the vertebrae which is the case of ours, less frequently in between the oesophagus and the trachea and extremely rarely anterior to the trachea. Furthermore our cadaver featured type $\mathrm{H}$ of Adachi-Williams-Nakagawa classification of the aortic arch variations which occurs in average of $0.5 \%$ of Japanese population [1].

Non-recurrent laryngeal nerve was first reported by Stedman in 1823 [18]. The incidence of the right NRLN is $0.3-1.6 \%$ and that of the left NRLN is about $0.04 \%$ [3]. NRLN often accompanies abnormal blood vessel development and is closely related to the absence of the brachiocephalic trunk or ARSA. NRLN can be classified into three types: type 1 is near the superior thyroid artery, type 2 courses parallel to the inferior thyroid artery and is further divided into type
2A that passes anterior to the artery and type $2 \mathrm{~B}$ that runs in between the branches of the artery $[5,19]$. NRLN of this case belongs to type $2 \mathrm{~A}$.

As addressed by Kausel et al. [10], variations of the classical thoracic duct's configuration can be seen at its termination, course, number of ducts and the location of its tributaries. They studied the thoracic ducts variations from 50 postmortem specimens and classified them into five types. From their classification, the thoracic duct of the present study that drained into the right brachiocephalic vein belongs to type 1 with the incidence of $2 \%$.

\section{Embryological background}

Initially in the human embryo there exist six pairs of branchial arch arteries arising from the aortic sac during the development of aorta. The development of the primitive aortic arches into vessels is a complicated process which can often engender congenital anomalies [9]. Generally the proximal portion of the RSA would be formed from the right $4^{\text {th }}$ arch and the distal portion from the right dorsal aorta and the right $7^{\text {th }}$ intersegmental artery. However when the right $4^{\text {th }}$ arch is absent, the proximal portion of the RSA derives from the right $7^{\text {th }}$ intersegmental artery instead. And then the distal portion of artery forms from the continuing dorsal aortic root caudal to it. In such case the RSA becomes ARSA [7].

The variation of recurrent laryngeal nerve could be an aftereffect of ARSA. The $4^{\text {th }}$ and the $6^{\text {th }}$ primitive aortic arches take part in the development; the $5^{\text {th }}$ and the $6^{\text {th }}$ arch pulls the recurrent laryngeal nerve 
down and later regresses leaving the nerve to coil around the RSA derived from the $4^{\text {th }}$ arch [20]. As stated previously, however, the degeneration of the $4^{\text {th }}$ arch leads to the absence of the proximal portion of the RSA causing the recurrent laryngeal nerve to have no artery to coil around and to become NRLN that directly enters the larynx [2].

Embryologically, both the right and the left thoracic ducts exist along with numerous lymphatic channels anastomosing between the two ducts. Normally, the definitive thoracic duct is formed by the lower part of the right duct merging with the upper part of the left duct. A theory surrounding the development of the right thoracic duct postulates that the lower part of the left thoracic duct connects with the upper part of the right duct. This can be induced that ARSA, which crosses the superior mediastinum from left to right, physically blocks the thoracic duct from taking the normal course and deviate towards the right [17].

\section{Clinical importance}

Although ARSA is usually asymptomatic, $10 \%$ of the people with ARSA show tracheo-oesophageal symptoms such as dysphagia lusoria as a result of ARSA compressing the oesophagus [8]. ARSA may be related to the difficulty in swallowing and had reported patients suffering more as they age due to the decreasing flexibleness of the oesophagus and the vessels [16]. Younger patients with ARSA may have respiratory symptoms caused by compression on the trachea that is not yet rigid enough to withstand the push of the artery [6]. A more common symptom of ARSA is Kommerell's diverticulum, a dilation of the artery of the aortic arch that leads to aneurysm usually at the proximal part of ARSA. This incidence occurs in $60 \%$ of ARSA patients and treatments may be vital depending on the size of the dilation [12]. Furthermore upper limb ischaemia due to the compression of ARSA between the oesophagus and the vertebrae is reported to be $30-45 \%$ of the patients hence appropriate reconstruction is necessary [13].

Non-recurrent laryngeal nerve is also a notable anomaly that must be examined especially when operating a thyroid or parathyroid surgery. The incidence of neural damage during surgeries of the recurrent laryngeal nerve is $0.5-2 \%$ whereas that of NRLN is around $12.9 \%$ being six times more vulnerable to damage $[5,11]$. For this reason, NRLN is a challenging complication in surgeries and extra caution is crucial.
The abnormal course of the thoracic duct may result in inadvertent injury to the duct especially during oesophagectomy, subsequently causing chylothorax with the iatrogenic occurrence of $3 \%$. Chylothorax, a scarce condition from the thoracic duct injury, causes chyle leakage to the pleural space and leads to respiratory distress, malnutrition and immunosuppression that can be fatal to patients [15].

The present study examines concurrent occurrence of ARSA, NRLN and the right thoracic duct. Despite some case reports on ARSA, there are only two reports on the simultaneous instance of these three anomalies $[14,15]$. Considering the insufficiency of such reports and the rareness, one might not acknowledge the possibility of the accompanying three variations. Thus this case report alerts the possibility of simultaneous instance of ARSA, NRLN and the right thoracic duct to the anatomical and the clinical fields.

\section{Acknowledgements}

This work was supported by ETRI R\&D Program [15ZC1400, The Development of a Realistic Surgery Rehearsal System based on Patient Specific Surgical Planning] funded by the Government of Korea.

\section{REFERENCES}

1. Adachi B (1928) Das Arteriensystem der Japener. Kenkyusha Press, Tokyo.

2. Avisse $C$, Marcus $C$, Delattre JF, Marcus $C$, Cailliez-Tomasi JP, Palot JP, Ladam-Marcus V, Menanteau B, Flament JB (1998) Right nonrecurrent inferior laryngeal nerve and arteria lusoria: the diagnostic and therapeutic implications of an anatomic anomaly. Review of 17 cases. Surg Radiol Anat, 20: 227-232. doi: 10.1007/s00276-998-0227-7.

3. Buła G, Mucha R, Paliga M, Koziołek H, Niedzielski Z, Gawrychowski J (2015) Non-recurrent laryngeal nerve. Pol Przegl Chir, 87: 336-339. doi: 10.1515/pjs-2015-0066.

4. Epstein DA, Debord JR (2002) Abnormalities associated with aberrant right subclavian arteries-a case report. Vasc Endovascular Surg, 36: 297-303. doi: $10.1177 / 153857440203600408$.

5. Hong KH, Yoon HW, Cho YS (1998) A case of non-recurrent laryngeal nerve. Korean J Otolaryngol, 41: 1213-1215.

6. Janssen M, Baggen MGA, Veen HF, Smout AJ, Bekkers JA, Jonkman JG, Ouwendijk RJ (2000) Dysphagia lusoria: clinical aspects, manometric findings, diagnosis and therapy. Am J Gastroenterol, 95: 1411-1416. doi: 10.1111/j.15720241.2000.02071.x.

7. Kanaskar N, Vatsalaswamy P, Sonje P, Paranjape V (2014) Retroesophageal right subclavian artery. Adv Anat, 1-3. Article ID 934825. doi: 10.1155/2014/934825.

8. Ka-tak W, Lam WW, Yu SC (2007) MDCT of an aberrant right subclavian artery and of bilateral vertebral arteries with anomalous origins. Am J Roentgenol, 188: W274W275. doi:10.2214/ajr.05.0694. 
9. Kau T, Sinzig M, Gasser J, Lesnik G, Rabitsch E, Celedin S, Eicher W, Illiasch H, Hausergger KA (2007) Aortic development and anomailie. Seminars in Interventional Radiology, 24: 141-152. doi: 10.1055/s-2007-980040.

10. Kausel HW, Reeve TS, Stein AA, Alley RD, Stranahan A (1957) Anatomic and pathologic studies of the thoracic duct. J Thorac Surg, 34: 631-641. doi: 10.1016/s00034975(10)66611-8.

11. Lee EJ, Kong IS, Cho YJ, Yoo SH, Lee KS, Lee JM, Kim YW, Yang YS, Hong KH (2009) Non-recurrent inferior laryngeal nerve in thyroid surgery: the usefulness of preoperative neck CT and clinical review. Korean J Otolaryngol, 52: 828-832. doi: 10.3342/kjorl-hns.2009.52.10.828

12. Lee JH, Ko YG, Yoon YN, Choi D, Lee DY (2013) Hybrid treatment for thoracic aortic aneurysm combined with aberrant right subclavian artery. Korean Circ J, 43: 66-69. doi: 10.4070/kcj.2013.43.1.66.

13. Lin Y, Li M, Liu X (2013) Aberrant right subclavian artery in video-assisted thoracoscopic esophagectomy. J Thoracic Disease, 5: E137-E139. doi: 10.3978/j.issn.20721439.2013.07.21.

14. Nathan H, Gitlin G (1968) Thoracic duct terminating on the right side associated with aberrant retro-oesophagal right subclavian artery and truncus bicaroticus. Thorax, 23: 266-270. doi: 10.1136/thx.23.3.266.

15. Pena E, Zuniga J, Baena G (2013) Simultaneous occurrence of three anatomical variations: anomalous right subclavian artery, non-recurrent inferior laryngeal nerve and right thoracic duct. Int J Morphol, 31: 1181-1184. doi: 10.4067/s0717-95022013000400006.

16. Quain R (1844) Anatomy of the arteries of the human body. Taylor and Walton, London 152-155.

17. Srinivasarao $Y$, Thirupathi rao V, Chandana CS, S. Swayam JDR, Bapuji P, Kannan M (2013) Anatomical variations of the termination of the thoracic duct in humans. Int J Med Health Sci, 2: 230-234.

18. Stedman GW (1823) A singular distribution of the nerves and arteries of the neck and the top of the thorax. Edinb Med Surg J, 19: 564-565.

19. Yetişir F, Özkardeş $A B$, Dündar HZ, Birkan B, Çiftci $A B$, Kılıç M. (2014) Non-recurrent laryngeal nerve. Ulusal Cer Derg, 30: 112-114. doi: 10.5152/ucd.2013.22.

20. Yetisir F, Salman AE, Çiftçi B, Teber A, Kiliç M (2012) Efficacy of ultrasonography in identification of non-recurrent laryngeal nerve. Int J Surg, 10: 506-509. doi: 10.1016/j. ijsu.2012.07.006. 